\title{
Compressed Sensing, Sparsity, and the Reliability of Tomographic Reconstructions
}

\author{
Yi Jiang ${ }^{1}$, Robert Hovden², David A. Muller ${ }^{2,3}$, Veit Elser ${ }^{1}$ \\ 1. Department of Physics, Cornell University, Ithaca, NY 14853 \\ 2. School of Applied and Engineering Physics, Cornell University, Ithaca, NY 14853 \\ 3. Kavli Institute at Cornell for Nanoscale Science, Ithaca, New York 14853
}

Motivated by compressed sensing [1,2], sparsity-exploiting 3D image reconstruction methods have been introduced to electron tomography $[3,4]$. These optimization-based methods utilize prior knowledge to provide improved reconstruction quality with reduced sampling. Although several studies using both experimental and simulated data showed positive results [3,4], we still lack a fundamental understanding of how and when these algorithms fail. Traditional reconstructions, such as weighted back projection, have predictable and well-understood artifacts. With algorithms that seek sparse solutions, the artifacts and failure are often more insidious - cleverly mimicking specimen structures. Adopting these new algorithms into routine tomography requires thorough investigation.

Here, we use computer simulations to quantify the number of measurements required for accurate tomographic reconstruction. Inspired by the work of Jörgensen [5], we study the performance of sparsity-exploiting methods as a function of the percentage of non-zero pixels $(k)$ and relative sampling $(\mu)$, which is defined as the ratio of the number of projections to the sufficient projection number. The sufficient projection number is the smallest number of projections that gives a full rank measurement matrix and is often sufficient for traditional reconstruction methods. For each pair of $(k, \mu)$, we generate 100 semi-realistic test images (of similar features) and report the percentage of "accurate" reconstruction whose root-mean-square error is less than 0.05 .

Figure 1 shows the simulation results using noise free data and the basis pursuit (BP) method-a sparsity-exploiting method that minimizes the $L_{1}$ norm of the image [6]. For complex objects with a large percentage of non-zero pixels, the reconstruction fails with small amount of measurements (black region). Even sparse objects of modest complexity contain many artifacts without sufficient information (Fig. 1,j,k). However, with more tilt projections, complete and accurate reconstruction is possible (Fig. $1, i)$. The sharp transition from inaccurate to accurate reconstruction regimes enables us to quantitatively predict the least number of measurements suffices for reconstructing different types of specimens. The fact that the transition boundary is below 1.0 is confirmation that the sparsity-exploiting method can outperform traditional methods in ideal situations.

The results in Figure 1 set a benchmark for studying more realistic systems. As shown in Figure 2 (left), the sparsity-exploiting method is highly sensitive to noise. Even with high signal-to-noise data, the region of accurate reconstruction is significantly reduced. Moreover, Figure 2 (right) reveals that the "missing wedge" problem also shifts up the transition boundary. The deviation is relative small at $\pm 70^{\circ}$ tilt range, but the method has very limited success when the tilt range is below $\pm 50^{\circ}$.

[1] E.J. Candès, J. Romberg, T. Tao, IEEE Trans. Inform. Theory 52 (2006), p. 489-509.

[2] D. L. Donoho, IEEE Trans. Inform. Theory 52 (2006), p. 1289-1306.

[3] Z. Saghi et al, Nano letters, 11 (2011), p. 4666-4673.

[4] B. Goris et al, Ultramicroscopy 113 (2012), p. 120-130. 
[5] J. S. Jörgensen et al, SIAM J. on Scientific Computing, Submitted.

[6] E. van den Berg et al, SIAM J. on Scientific Computing 31 (2012), p. 890-912.
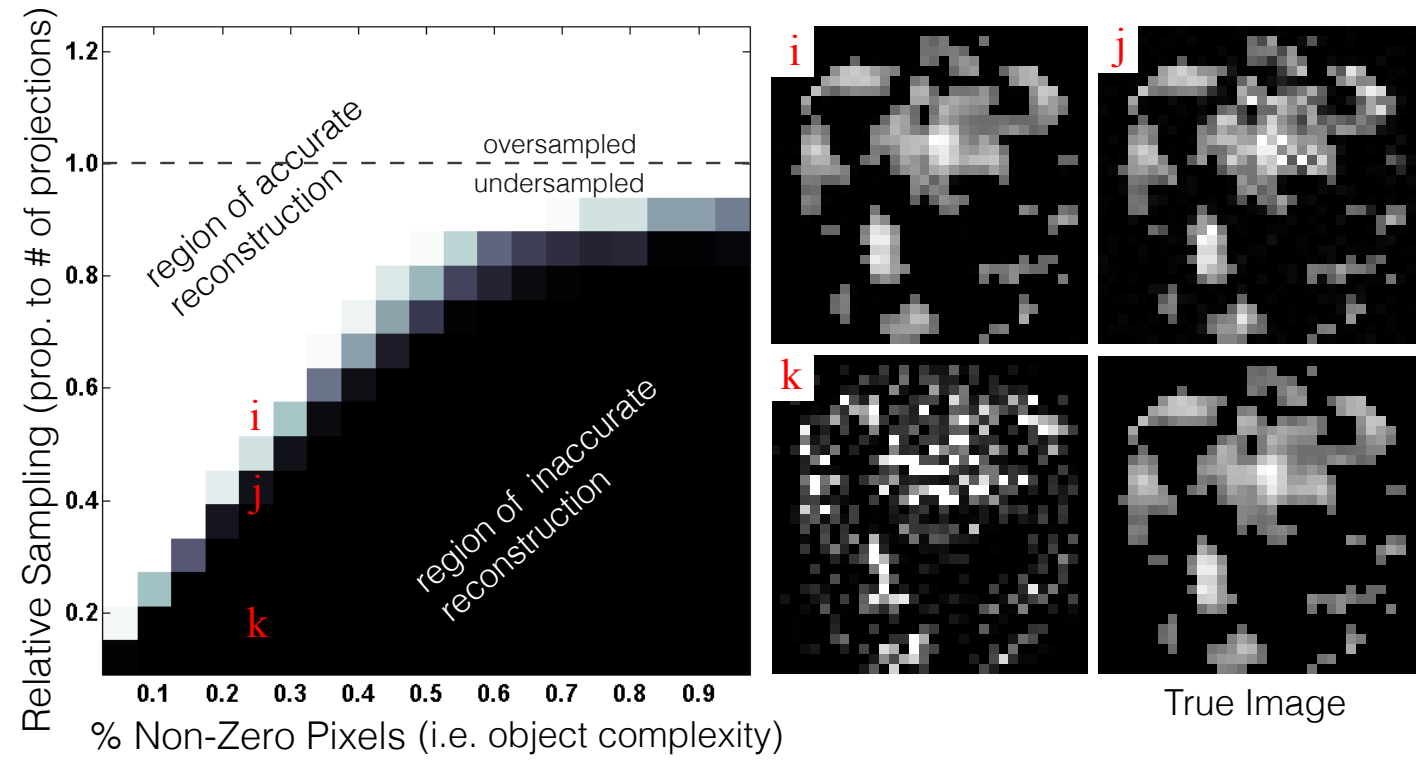

True Image

Figure 1: (Left) Percentage of accurate reconstructions of the basis pursuit method. There is a sharp transition from the region where all the test images are accurately reconstructed to the region of zero recovery rates. (Right) Three reconstructions with different number of projections. In the region of accurate reconstruction, the reconstruction (i) matches the true image. As relative sampling decreases, noticeable artifacts start to appear $(\mathrm{j}, \mathrm{k})$.
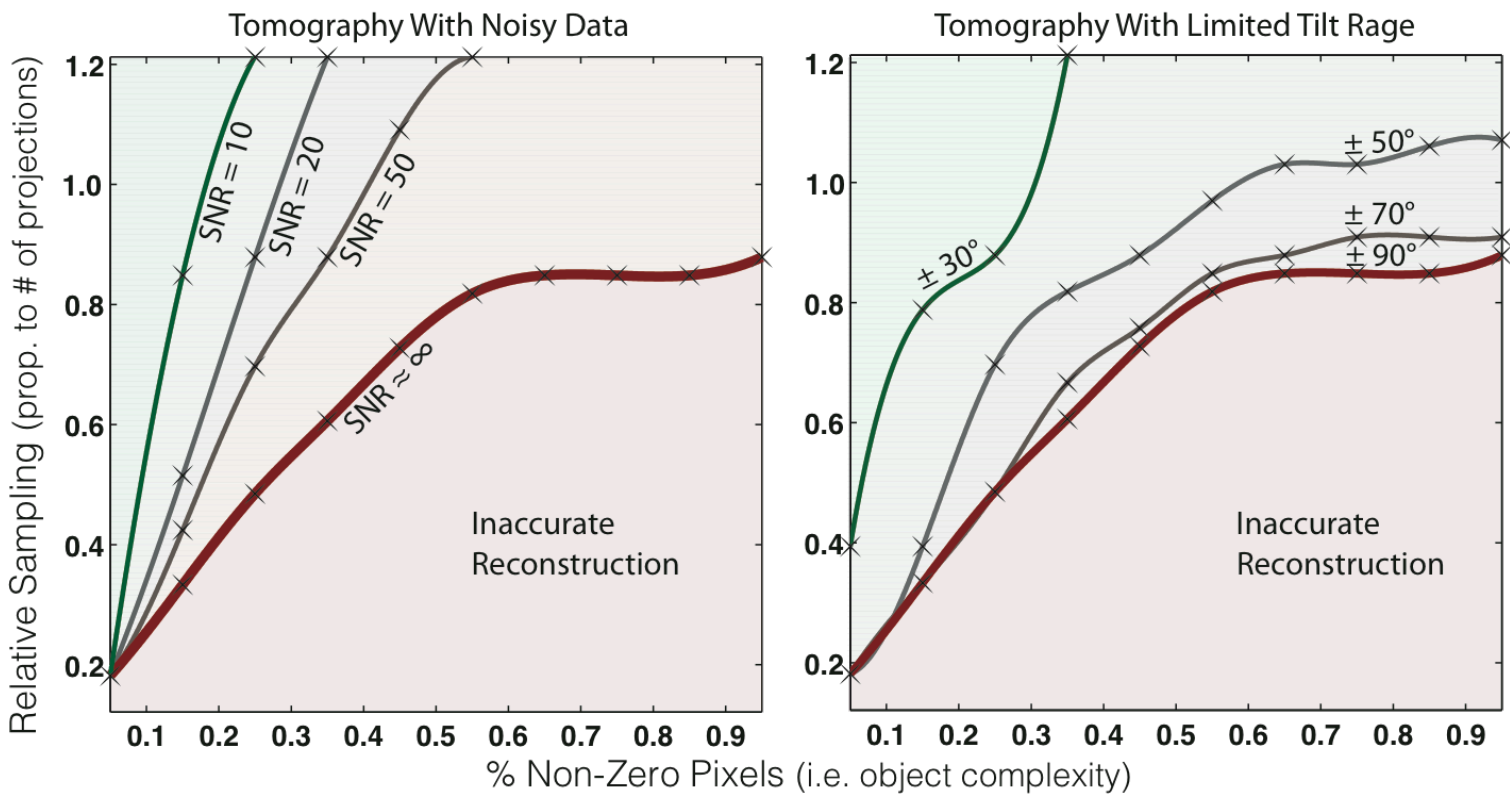

Figure 2: (Left) Transition boundaries of noisy data for different signal-to-noise ratios. Adding noise greatly reduces the region of accurate reconstruction. (Right) Transition boundaries vs. tilt range. The boundary for $\mathrm{a} \pm 70^{\circ}$ tilt range is close to that of the full tilt range, but the method has very limited success when the tilt range is below $\pm 50^{\circ}$. 